\title{
Infrared gluons, intrinsic transverse momentum and rising total cross-sections *
}

\author{
A. Grau ${ }^{\mathrm{a}}$, R. M. Godbole ${ }^{\mathrm{b}}$, G. Pancheri $^{\mathrm{c}}$ and Y. N. Srivastava ${ }^{\mathrm{d}}$ \\ ${ }^{a}$ Departamento de Fisica Teorica y del Cosmos, Universidad de Granada, Spain \\ ${ }^{\mathrm{b}}$ Centre for High Energy Physics, Indian Institute of Science, Bangalore, 560012, India \\ ${ }^{\mathrm{c}}$ INFN Frascati National Laboratories, Frascati, I00444, Italy \\ dPhysics Department and INFN, University of Perugia, Perugia, Italy
}

We discuss the infrared limit for soft gluon $k_{t}$-resummation and relate it to physical observables such as the intrinsic transverse momentum and the high energy limit of total cross-sections.

\section{Introduction}

We present an analysis of the rise of total crosssections achieved in our eikonal mini-jet model through an infrared (IR) singular $\alpha_{s}$ and soft gluon resummation [1]. We call it the BlochNordsieck (BN) model because in it we include an infinite number of independently emitted very soft IR gluons. Consider hadron-hadron scattering at a c.m.s energy $\sqrt{s}$. In the eikonal representation, the elastic and the total cross-sections can be written as

$\sigma_{\text {elastic }}=\int d^{2} \mathbf{b}\left|1-e^{i \chi(b, s)}\right|^{2}$,

$\sigma_{\text {total }}=2 \int d^{2} \mathbf{b}\left[1-e^{-\mathcal{I} m \chi(b, s)} \cos \Re e \chi(b, s)\right]$.

The above two equations give for the total inelastic cross-section

$\sigma_{\text {total inelastic }} \equiv \sigma_{\text {inel }}=\int d^{2} \mathbf{b}\left[1-e^{-2 \mathcal{I} m \chi(b, s)}\right]$

*Presented at Hadron Structure '09, Tatranská Štrba, September 2009. Thanks are due to the Center for Theoretical Physics of the Massachusetts Institute of Technology, for hospitality while this work was being written. R. G. acknowledges support from Department of Science and Technology, India for financial support under Grant No. SR/S2/JCB-64/2007 (J. C. Bose fellowship) This work has been partially supported by MEC (FPA2006-05294) and Junta de Andalucía (FQM 101 and FQM 437).
By relating the inelastic cross-section to the probability of all possible inelastic processes, considered independent of one another, one relates the average number of inelastic collisions to the imaginary part of the eikonal function, and the task is to adequately model this number. Neglecting the real part of the eikonal (a good approximation for hadronic cross-sections at high energies), we obtain a simplified expression for the total crosssection

$\sigma_{\text {total }}=2 \int d^{2} \mathbf{b}\left[1-e^{-\bar{n}(b, s) / 2}\right]$.

Some time ago, it was noticed $[2,3]$ that perturbative QCD provides a simple mechanism for rising total cross-sections. The average number of collisions $\bar{n}(b, s)$ increases because of the increasing number of low $x$ gluon-gluon collisions. These can be calculated perturbatively for all parton-parton processes with outgoing partons of $p_{t}>p_{t, \text { min }}$.

The cut-off $p_{t, \min }$ performs a double role: (i) it avoids the Rutherford singularity as $p_{t} \rightarrow 0$, as well as (ii) provides a scale above which perturbative parton-parton cross-section estimates can be made using the asymptotic freedom $(\mathrm{AF})$ expression for the strong coupling constant $\alpha_{s}$.

For the complete $\bar{n}(b, s)$, we need to add a non- 
perturbative part

$\bar{n}(b, s)=n_{N P}(b, s)+n_{\text {hard }}(b, s)$,

where the non perturbative (NP) term parametrizes the contribution of all those processes for which initial partons scatter with $p_{t}<p_{t, \text { min }}$. We approximate the hard term, which is responsible for the high-energy rise and which we expect to dominate in the extremely high energy limit, as

$n_{\text {hard }}(b, s)=A(b, s) \sigma_{\text {jet }}(s)$

and calculate $\sigma_{j e t}(s)$ using LO proton-proton cross-sections obtained from parton cross-sections and DGLAP [4] evoluted Parton Density Functions of current use [5] at the scale $Q^{2}=p_{t}^{2}$. These cross-sections, when $p_{t, \text { min }} \approx 1 \div 2 \mathrm{GeV}$, have been called mini-jets to distinguish them from the high- $p_{t}$ jet cross-sections which are experimentally visible at high energies. These mini-jet cross-sections grow too much rapidly with energy. Imposition of unitarity in the eikonal impact parameter representation does indeed dampen in part this unacceptable growth.

However, in order to properly reproduce the observed total cross-section high energy rise, from $\sqrt{s} \approx 10 \div 20 \mathrm{GeV}$ to the Tevatron, Cosmic rays and extrapolations beyond, one needs to properly model the impact parameter dependence of partons in the hadrons. We shall discuss this in the coming sections.

\section{Revisiting $k_{t}$ resummation}

\subsection{The infrared limit in eikonal mini-jet models with soft gluon resummation}

In our eikonal model for the total cross-section, the rise is driven by perturbative QCD scattering tempered by soft gluon resummation down to IR region [1]. To perform resummation in this region, we used an expression for the effective quark-gluon interaction as $k_{t}^{\text {gluon }} \rightarrow 0$ given by

$\alpha_{s}\left(k_{t}^{2}\right)=\frac{p}{b_{0} \ln \left[1+p\left(\frac{k_{t}^{2}}{\Lambda^{2}}\right)^{p}\right]}$

where $b_{0}=\left(33-2 N_{f}\right) / 12 \pi$ is the one-loop coefficient of the QCD beta function, and $\Lambda$ is the QCD scale. In the $k_{t}^{2} \gg \Lambda^{2}$ limit, the above expression reduces to the usual one loop asymptotic freedom expression for $\alpha_{s}$, whereas $k_{t}^{2}<\Lambda^{2}$ limit allows integration into the IR region, provided $p<1$. In the above expression, the constant $p$ in front of $k^{2} / \Lambda^{2}$ was included to ensure that $\alpha_{s} \neq 0$ in the limit $p \rightarrow 0$. However, in [6] we have subsequently found that in this model, one needs $p>1 / 2$ for analyticity of the scattering amplitude, so that the $p$ going to zero limit is never of interest, and one could as well use, for interpolation between the IR and the UV region, a simpler expression

$$
\begin{aligned}
\alpha_{s}\left(k_{t}^{2}\right)= & \frac{p}{b_{0} \ln \left[1+\left(\frac{k_{t}^{2}}{\Lambda^{2}}\right)^{p}\right]} \\
& \rightarrow \frac{1}{b_{0} \ln \left[\frac{k_{t}^{2}}{\Lambda^{2}}\right]} \quad \text { for } \quad \frac{k_{t}^{2}}{\Lambda^{2}} \gg 1 \\
& \rightarrow \frac{p}{b_{0}}\left(\frac{k_{t}^{2}}{\Lambda^{2}}\right)^{p} \quad \text { for } \frac{k_{t}^{2}}{\Lambda^{2}} \ll 1
\end{aligned}
$$

\subsection{The impact parameter distribution}

In our BN model, we have proposed the impact parameter distribution of partons in hadrons to be described by the Fourier transform of the soft gluon transverse momentum distribution, namely, for the average number of hard collisions, $n_{\text {hard }}(b, s)$, we have put

$$
\begin{aligned}
n_{\text {hard }}(b, s) & =\sum_{i, j} \int \frac{d x_{1}}{x_{1}} \\
& \times \int \frac{d x_{2}}{x_{2}} f_{i / a}\left(x_{1}, p_{t}^{2}\right) f_{j / b}\left(x_{2}, p_{t}^{2}\right) \\
& \times \int d z \int d p_{t}^{2} A_{B N}(b, M) \frac{d \sigma}{d p_{t}^{2} d z}
\end{aligned}
$$

where $f_{i / a}\left(x, p_{t}^{2}\right)$ are the parton densities in the colliding hadrons $a$ and $b$, evolved at the scale $p_{t}^{2}, z=\hat{s}_{j e t} /\left(s x_{1} x_{2}\right)$, with $\sqrt{\hat{s}_{j e t}}$ being the invariant mass of the final parton-parton system emerging as two jets and $\frac{d \sigma}{d p_{t}^{2} d z}$ is the differential cross-section for the process

parton + parton $\rightarrow$ jet jet $+X$

The impact distribution function corresponding to the partonic collision is assumed to be given by

$A_{B N}(b, M)=A_{0} e^{-h(b, M)}$ 
with the normalization constant $A_{0}$

$$
A_{0}=\frac{1}{2 \pi \int b d b e^{-h(b, M)}}
$$

and

$$
\begin{aligned}
h(b, M)= & \frac{2 c_{F}}{\pi} \int_{0}^{M} \frac{d k_{\perp}}{k_{\perp}} \alpha_{s}\left(\frac{k_{\perp}^{2}}{\Lambda^{2}}\right) \\
& \times \ln \frac{M+\sqrt{M^{2}-k_{\perp}^{2}}}{M-\sqrt{M^{2}-k_{\perp}^{2}}}\left[1-J_{0}\left(k_{\perp} b\right)\right] .
\end{aligned}
$$

$M \equiv M\left(x_{1}, x_{2}, Q^{2}, s\right)$ is the maximum transverse momentum allowed to single gluon emission by the kinematics of the process

$\operatorname{parton}\left(x_{1}\right)+\operatorname{parton}\left(x_{2}\right) \rightarrow X\left(Q^{2}\right)+\operatorname{gluon}(k)$,

where X represents a particle system with invariant mass $Q^{2}$, i.e., two jets, for high energy parton scattering at LO. The kinematics of the above process gives [7]

$M\left(x_{1}, x_{2} ; Q^{2}, s\right)=\frac{\sqrt{\hat{s}}}{2}\left(1-\frac{Q^{2}}{\sqrt{\hat{s}}}\right)$,

with $\hat{s}=4 x_{1} x_{2} s$. We simplify the application of Eq. (11) by evaluating $A_{B N}(b, M)$ at a value $q_{\max }$ which represents the average of $\mathrm{M}$ over all parton-parton processes, namely we shall use from now on, the factorized expression

$$
n_{\text {hard }}(b, s)=A_{B N}(b, s) \sigma_{\text {jet }}\left(s, p_{t, \text { min }}\right)
$$

where the $s$-dependence of $A_{B N}(b, s)$ is obtained through

$$
\begin{aligned}
& \left\langle M\left(x_{1}, x_{2} ; Q^{2}, s\right)\right\rangle \equiv q_{\max }(s)=\frac{\sqrt{s}}{2} \times \\
& \frac{\sum_{i, j} \int \frac{d x_{1}}{x_{1}} f_{i / a}\left(x_{1}\right) \int \frac{d x_{2}}{x_{2}} f_{j / b}\left(x_{2}\right) \sqrt{x_{1} x_{2}} \int d z(1-z)}{\sum_{i, j} \int \frac{d x_{1}}{x_{1}} f_{i / a}\left(x_{1}\right) \int \frac{d x_{2}}{x_{2}} f_{j / b}\left(x_{2}\right) \int(d z)}
\end{aligned}
$$

with the lower limit of integration in the variable $z$ given by $z_{\text {min }}=4 p_{t, \text { min }}^{2} /\left(s x_{1} x_{2}\right)$. The scale parameter $q_{\max }$ is a slowly varying function of $\sqrt{s}$ which depends on the PDF's used. In any phenomenological application, the PDF's used to evaluate $q_{\max }$ will of course be of the same type as those used to evaluate $\sigma_{j e t}$. Notice that $q_{\max }$ is of the order of $p_{t, \text { min }}$, since most of the parton-parton cross-section is peaked at $p_{t, \min }$. In Eq. (19), we have dropped for simplicity the scale $p_{t}^{2}$ at which the densities are evaluated, but it is understood that all the densities are actually DGLAP evoluted. It is through $q_{\max }$ that $A_{B N}(b, s)$ acquires its energy dependence. This happens both from $e^{-h(b, s)}$ as well as through the normalization constant $A_{0}$. We shall show how $A_{0}$ depends on the energy in a later section.

The function $A_{B N}$ is obtained from the Fourier transform, $\mathcal{F}_{\mathcal{B N}}\left(K_{\perp}\right)$ of the transverse momentum distribution of the overall soft gluon radiation emitted (to LO) by quarks as the hadron breaks up because of the collision. This distribution is obtained by summing soft gluons to all orders, with a technique amply discussed in the literature [8-10], namely

$\mathcal{F}_{\mathcal{B N}}\left(K_{\perp}\right)=\frac{1}{2 \pi} \int b d b J_{0}\left(b K_{\perp}\right) e^{-h(b, M)}$

As discussed in [1], we use Eq. (20) with the soft gluon integration in Eq. (15) extended well below the QCD scale $\Lambda$, where the asymptotic freedom expression for $\alpha_{s}$ is not valid. We enter this region, through the expression in Eq. (7). In coordinate space, this $\alpha_{s}$ corresponds to a confining one-gluon exchange potential since it grows for large separation between quarks.

Using such an expression allows us to push the $k_{t}$-integration in Eq. (15) down to zero values and hence access the very large distances which are relevant to physical observables like the total cross-section or the intrinsic transverse momentum.

We now recall how Eq. (15) is commonly used. That is, one usually separates the IR region from the perturbative one as follows

$$
h(b, E)=c_{0}(\mu, b, E)+\Delta h(b, E),
$$

where

$$
\begin{aligned}
& \Delta h(b, E)= \\
& \frac{16}{3} \int_{\mu}^{E} \frac{\alpha_{s}\left(k_{t}^{2}\right)}{\pi}\left[1-J_{o}\left(b k_{t}\right)\right] \frac{d k_{t}}{k_{t}} \ln \frac{2 E}{k_{t}} .
\end{aligned}
$$


Since the integral in $\Delta h(b, E)$ now extends down to a scale $\mu \neq 0$, for $\mu>\Lambda_{Q C D}$ one can use the asymptotic freedom expression for $\alpha_{s}\left(k_{t}^{2}\right)$. Furthermore, having excluded the zero momentum region from the integration, $J_{o}\left(b k_{t}\right)$ is assumed to oscillate to zero and neglected. The integral of Eq. (22) is now independent of $b$ and can be performed, giving

$\Delta h(b, E)=\frac{32}{33-2 N_{f}}$

$\times\left\{\ln \left(\frac{2 E}{\Lambda}\right)\left[\ln \left(\ln \left(\frac{E}{\Lambda}\right)\right)-\ln \left(\ln \left(\frac{\mu}{\Lambda}\right)\right)\right]-\ln \left(\frac{E}{\mu}\right)\right\}$.

$\Lambda$ being the scale in the one-loop expression for $\alpha_{s}$. In the range $1 / E<b<1 / \Lambda$ the effective $h_{e f f}(b, E)$ is obtained by setting $\mu=1 / b[10]$. This choice of the scale introduces a cut-off in impact parameter space which is stronger than any power, since the radiation function, for $N_{f}=$ 4 , is now [10]

$e^{-h_{e f f}(b, E)}=\left[\frac{\ln \left(1 / b^{2} \Lambda^{2}\right)}{\ln \left(E^{2} / \Lambda^{2}\right)}\right]^{(16 / 25) \ln \left(E^{2} / \Lambda^{2}\right)}$.

Under the assumption that there is no physical singularity in the range of integration $0 \leq k_{t} \leq$ $1 / b$, the remaining $b$-dependent term, namely $\exp \left[-c_{0}(\mu, b, E)\right]$, is then dropped.

By contrast, it is the inclusion of the IR gluons, fortified by a singular but integrable $\alpha_{s}$, which shows up in our calculation as an energy independent smearing function phenomenologically called the intrinsic transverse momentum of partons. The connection between $c_{0}(\mu, b, E)$ and the intrinsic transverse momentum of partons is easily established formally if, in the region $b E \gg 1$, one makes the approximation $[11,12]$

$h(b, E) \approx b^{2} A$

with

$A=\frac{c_{F}}{4 \pi} \int d k^{2} \alpha_{s}\left(\frac{k^{2}}{\Lambda^{2}}\right) \ln \frac{4 E^{2}}{k^{2}}$.

We obtain a function $h(b, E)$ which, through Eq. (13), gives a gaussian fall-off as in models where $A(b)$ is the Fourier transform of an intrinsic transverse momentum distribution of partons, i.e. $\exp \left(-k_{\perp}^{2} / 4 A^{2}\right)$. We shall discuss this point further in the next section.

\section{The intrinsic transverse momentum}

The intrinsic transverse momentum is a phenomenological description of the very low- $p_{t}$ behaviour of hadrons, Drell-Yan pairs, $W$-mesons, jet-pairs, etc., produced in hadronic collisions. It was discussed in [7,9-11] and recently has been studied phenomenologically in [13]. It reflects the existence of a residual non-collinearity of quarks in the colliding hadrons, which cannot be estimated perturbatively through the Sudakov form factor. Writing the contribution of the intrinsic transverse momentum as $\exp \left(-\frac{k_{\perp}^{2}}{<k_{t}^{2}>}\right)$ and comparing with Eq. (25) we have $p_{t-\text { intrinsic }}=$ $\sqrt{\left\langle k_{t}^{2}\right\rangle}=2 \sqrt{A}$. In our model, in order to estimate a value for the intrinsic transverse momentum as a function of energy as done in [12], the integration in the soft gluon momentum is pushed down to zero, using the singular but integrable expression for $\alpha_{s}$ presented in Sect. 2. In Fig. 1. the value of $p_{t-i n t r i n s i c}$ from [12] is plotted as a function of $M$, using $p=5 / 6$ following the argument in [14] about linearly rising Regge trajectories.

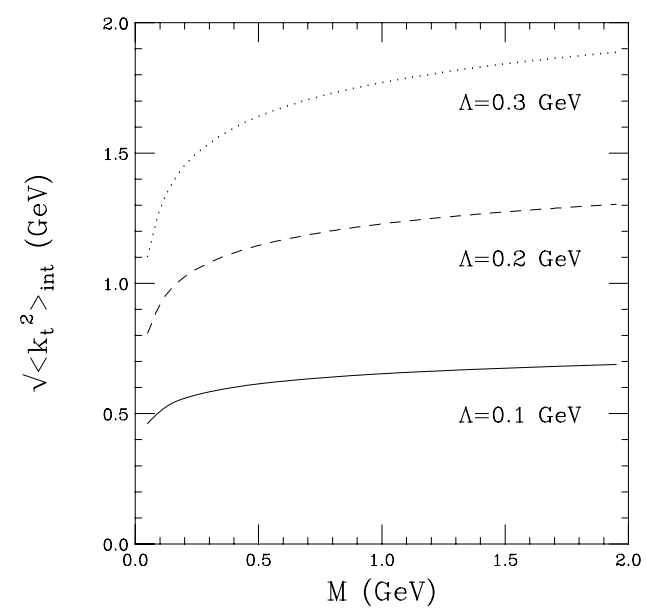

Figure 1. Intrinsic transverse momentum as a function of $M$ for different values of $\Lambda, p=5 / 6$.

In Fig. 2 from [12] we reproduce the function $A_{B N}(b, s)$ for a range of values of the scale $\mathrm{M}$, 
called here $q_{\max }$. We also compare our proposed expression with the result for the form factor model, in which the impact factor is independent of energy and obtained through the convolution of the form factors of the colliding hadrons, namely

$A_{F F}(b)=\frac{1}{(2 \pi)^{2}} \int d^{2} \vec{q} e^{i b \cdot q} \mathcal{F}_{1}(q) \mathcal{F}_{2}(q)$.

For protons, the usual parametrization

$\mathcal{F}_{\text {proton }}(q)=\left(\frac{\nu^{2}}{q^{2}+\nu^{2}}\right)^{2}, \quad \nu^{2}=0.71 \mathrm{GeV}^{2}$,

leads to the following expression for the overlap function

$A_{F F}(b)=\frac{\nu^{2}}{96 \pi}(\nu b)^{3} K_{3}(\nu b)$.

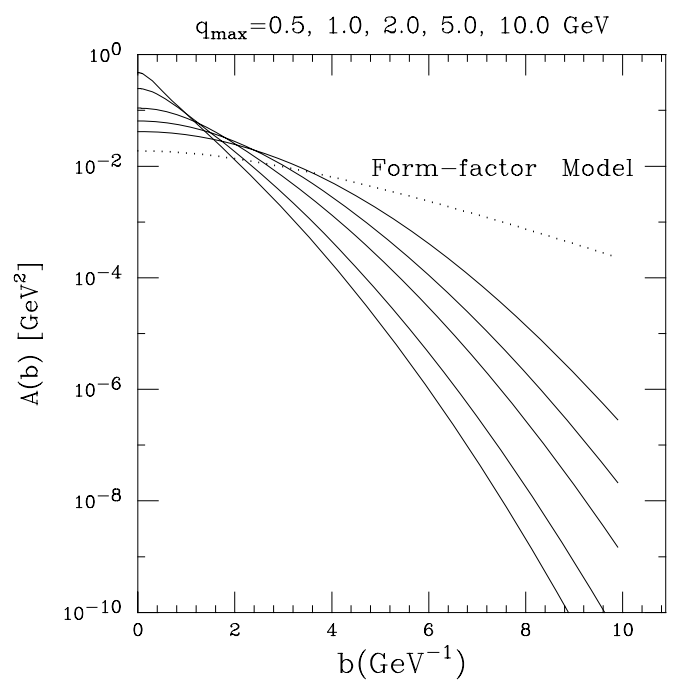

Figure 2. The $\mathrm{A}(\mathrm{b})$ distribution function from the Bloch-Nordsieck model for different $q_{\max }$ values compared with the form factor model $A_{F F}(b)$, $p=5 / 6$.

The expression for $\alpha_{s}$ in Eq. (7) allows to extend soft gluon resummation into the ultra-soft, zero momentum region. This can be done numerically, since it is not possible to obtain an analytic expression for $h(b, M, \Lambda)$ valid in the full integration region. One can however divide the integration region in various intervals, and make suitable approximations for the integrand. Thus a study of the $b$-region of interest, gives the result discussed in [6], namely

$$
b>\frac{1}{N_{p} \Lambda}>\frac{1}{M}
$$

$$
\begin{aligned}
& h(b, M)=\frac{2 c_{F}}{\pi}\left[\bar{b} \frac{b^{2} \Lambda^{2 p}}{2} \int_{0}^{\frac{1}{b}} \frac{d k}{k^{2 p-1}} \ln \frac{2 M}{k}\right. \\
& \left.+2 \bar{b} \Lambda^{2 p} \int_{\frac{1}{b}}^{N_{p} \Lambda} \frac{d k}{k^{2 p+1}} \ln \frac{M}{k}+\bar{b} \int_{N_{p} \Lambda}^{M} \frac{d k}{k} \frac{\ln \frac{M}{k}}{\ln \frac{k}{\Lambda}}\right] \\
& =\frac{2 c_{F}}{\pi}\left[\frac{\bar{b}}{8(1-p)}\left(b^{2} \Lambda^{2}\right)^{p}\left[2 \ln (2 M b)+\frac{1}{1-p}\right]\right. \\
& +\frac{\bar{b}}{2 p}\left(b^{2} \Lambda^{2}\right)^{p}\left[2 \ln (M b)-\frac{1}{p}\right] \\
& +\frac{\bar{b}}{2 p N_{p}^{2 p}}\left[-2 \ln \frac{M}{\Lambda N_{p}}+\frac{1}{p}\right] \\
& \left.+\bar{b} \ln \frac{M}{\Lambda}\left[\ln \frac{\ln \frac{M}{\Lambda}}{\ln N_{p}}-1+\frac{\ln N_{p}}{\ln \frac{M}{\Lambda}}\right]\right]
\end{aligned}
$$

where $c_{F}=4 / 3$ for emission from quark legs, $\bar{b}=12 \pi /\left(33-2 N_{f}\right)$, and $N_{p}=(1 / p)^{1 / 2 p}>1$ for $p<1$.

Through this approximation, we see that our ansatz for $\alpha_{s}$ for $k^{2} / \Lambda^{2} \ll 1$ leads to the sharp cut-off in $e^{-h(b, M)}$ at large- $b$ values which we shall exploit to study the very large energy behaviour of the total cross-section in our model, namely we obtain

$$
A_{B N}(b, s)=A_{0} e^{-h(b, M)} \approx e^{-(b \bar{\Lambda})^{2 p}}, b>\frac{1}{\Lambda}>\frac{1}{M}
$$

Eq. (32) is similar to Eq. (25) for $p \approx 1$. For the soft integral in $h(b, M)$ to be finite, however, $p<1$ as one can see from the actual expression 
one obtains from $\bar{\Lambda}$ through Eq. (31), namely

$$
\begin{aligned}
\bar{\Lambda}(b, s)= & \Lambda\left\{\frac { c _ { F } \overline { b } } { 4 \pi ( 1 - p ) } \left[\ln \left(2 q_{\max }(s) b\right)\right.\right. \\
& \left.\left.+\frac{1}{1-p}\right]\right\}^{1 / 2 p} .
\end{aligned}
$$

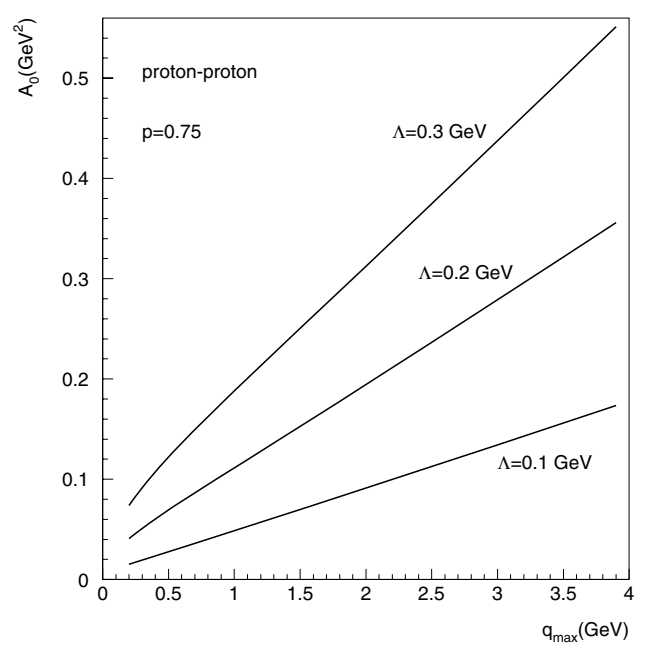

Figure 3. The normalization constant $A_{0}$ as a function of $q_{\max }$ for different values of $\Lambda$.

We plot in the next figures the values taken by $A_{0}$ as a function of energy for different cases. In Fig. 3 we show how $A_{0}$ varies as function of $q_{\max }$ for proton-proton, for different values of the QCD scale $\Lambda$. Notice that, following our recent phenomenological applications $[1,15]$, we have used $\Lambda=100 \mathrm{MeV}$ in the soft gluon integral, and $p \approx 0.75$. In Fig. 4 we show $A_{0}$ as a function of $\sqrt{s}$ for the two cases of proton-proton and $\gamma p$. The two cases differ because of different values of the scale parameter $q_{\max }$, which reflect different parton densities for photons and protons [15]. We also plot in Fig. (5) the energy dependence of $\bar{\Lambda}$, for proton-proton and for $\gamma p$, obtained through the parameter $q_{\max }$ as mentioned before.

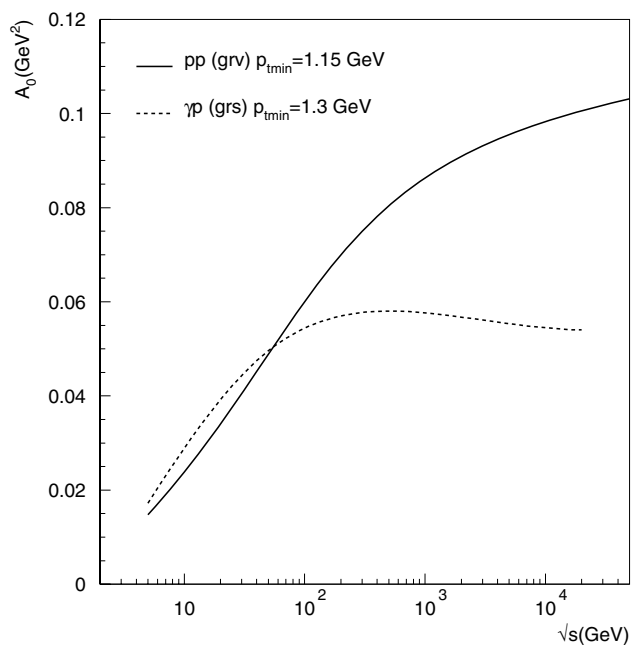

Figure 4. The normalization constant $A_{0}$ as a function of $\sqrt{s}$ for the case of proton-proton and $\gamma p$ total cross-section calculation.

\section{The Froissart limit}

The sharp falling off at very large $b$-values exhibited by our proposed impact parameter distribution can be exploited to discuss the large energy behaviour of the total hadronic cross-section in our model. Going to the very high energy limit in Eqs. $(4,5,18)$, we can write

$\sigma_{T}(s) \approx 2 \pi \int_{0}^{\infty} d b^{2}\left[1-e^{-n_{\text {hard }}(b, s) / 2}\right]$.

Inserting the asymptotic expression for $\sigma_{j e t}$ at high energies, which grows like a power of $s$, and $A_{B N}(b, s)$ from Eq. (32) we obtain

$n_{\text {hard }}=2 C(s) e^{-(b \bar{\Lambda})^{2 p}}$,

where $2 C(s)=A_{0}(s) \sigma_{1}\left(s / s_{0}\right)^{\varepsilon}$. The resulting expression for $\sigma_{T}$

$\sigma_{T}(s) \approx 2 \pi \int_{0}^{\infty} d b^{2}\left[1-e^{-C(s) e^{-(b \bar{\Lambda})^{2 p}}}\right]$

leads to

$\bar{\Lambda}^{2} \sigma_{T}(s) \approx\left(\frac{2 \pi}{p}\right) \int_{0}^{u_{0}} d u u^{\frac{1-p}{p}}=2 \pi u_{0}^{1 / p}$ 


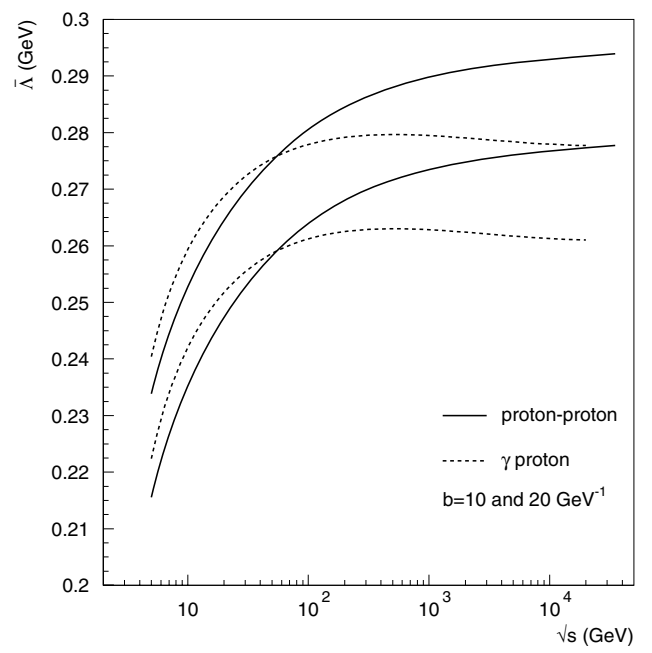

Figure 5. $\bar{\Lambda}$ as a function of energy, for photons and protons.

with

$u_{0}=\ln \left[\frac{C(s)}{\ln 2}\right] \approx \varepsilon \ln s$.

To leading terms in $\ln s$, we derive the asymptotic energy dependence

$\sigma_{T} \rightarrow[\varepsilon \ln (s)]^{(1 / p)}$.

Since $1 / 2<p<1$ [17], the above result shows that, with soft gluon momenta integrated into the IR region, $k_{t}<\Lambda$, and a singular but integrable coupling to the quark current, our model leads to satisfaction of the Froissart-Martin bound $[17,18]$. This region, with the scale $\Lambda \simeq \mathcal{O}\left(\Lambda_{Q C D}\right)$ is of course inaccessible to the perturbative coupling for $\alpha_{s}$, but it plays a crucial role in many inclusive low $p_{t}$ processes. One reason to neglect it could be that gluons with $\left|k_{\perp}\right| \ll \Lambda$ would see the hadron as a point-like object [19] and such emissions would have a small probability, because of colour screening. This argument is appealing, and similar to the one mentioned in Sect. 2, but in our opinion, there is no compelling theoretical reason to assume that ultrasoft gluon emission in high energy reactions has low probablity. This argument could be applied to an isolated hadron, but not to high energy hadronic scattering described through the scattering of partons, where soft gluon emission is stimulated by QCD interactions. It is through this interaction that we can expect the transition between hadrons and quarks to arise. A singularity in the infrared region would indeed provide a cut-off to separate quarks from hadrons and lead to such transition. This is the rationale behind going into the zero momentum region.

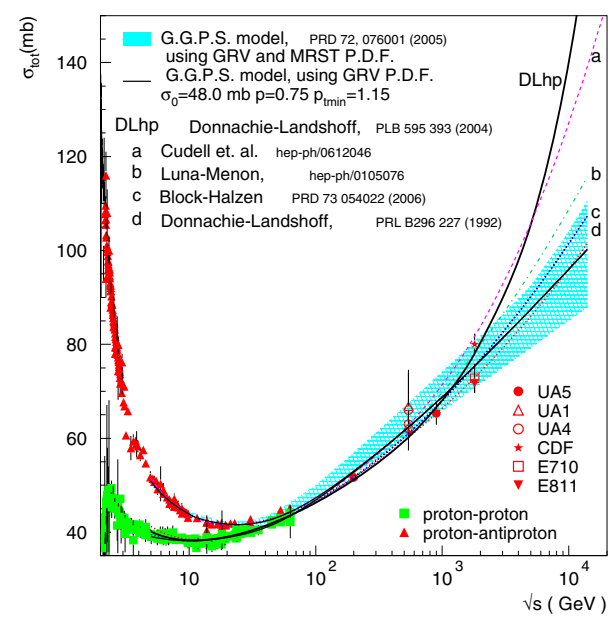

Figure 6. Data for the total proton-proton crosssection and comparison with the $\mathrm{BN}$ and other models [16].

We show in the last figure of this contribution a comparison between our model and the existing data for the total proton-proton cross-section. The band corresponds to a set of parameter values consistent with the discussion in the previous section, namely $p=0.75 \div 0.8, p_{t, \min }=1.15 \mathrm{GeV}$ and GRV and MRST [5] densities in the calculation of $\sigma_{j e t}$ and $q_{\max }$.

\section{Conclusions}

We have shown how a simple ansatz for the IR soft gluon spectrum allows to study the large impact parameter behaviour of some hadronic 
quantities, like the intrinsic transverse momentum and, most important, the total cross-section. Our ansatz relies on a power law behaviour for the coupling of very soft gluons to the quark current, which makes it possible to integrate the soft gluon spectrum into the IR region. Our expression for the coupling is singular but integrable and interpolates between the UV and the IR region.

\section{Acknowledgments}

Thanks are due to the Organizers for this very interesting Conference and for hospitality. G.P is also grateful to L. Lipatov for enlightening discussions.

\section{REFERENCES}

1. R.M. Godbole, A. Grau, G. Pancheri, Y.N. Srivastava,Phys.Rev.D72 $076001 \quad$ (2005); A. Achilli, R. Hegde, R. M. Godbole, A. Grau, G. Pancheri and Y. Srivastava, Phys. Lett. B 659 (2008) 137.

2. D. Cline, F. Halzen and J. Luthe, Phys. Rev. Lett. 31 (1973) 491; T. K. Gaisser, F. Halzen, Phys. Rev. Lett. 54 (1985) 1754.

3. L. V. Gribov, E.M. Levin, M. G. Ryskin, Phys. Rept. 100 (1983 )1.

4. Y. L. Dokshitzer, Sov. Phys. JETP 46 (1977) 641 [Zh. Eksp. Teor. Fiz.73 (1977) 1216]; V. N. Gribov and L. N. Lipatov, Yad. Fiz. 15 (1972) 781 [Sov. J. Nucl. Phys. 15 (1972) 438]; G. Altarelli and G. Parisi, Nucl. Phys. B126 (1977) 298.

5. M. Glück, E. Reya, and A. Vogt, Z. Phys. C53(1992) 127; Z. Phys. C67 (1995) 433; Eur. Phys. J. C5 (1998) 461; A. D. Martin, R. G. Roberts, W. J. Stirling, and R. S. Thorne, Phys. Lett. B531 (2002) 216; H. L. Lai, J. Botts, J. Huston, J. G. Morfin, J. F. Owens, Jian-wei Qiu, W. K. Tung, H. Weerts, Phys. Rev. D51 (1995) 4763.

6. A. Grau, R.M. Godbole, G. Pancheri and Y.N. Srivastava, Soft Gluon $k_{t^{-}}$ Resummation and the Froissart bound, e-Print: arXiv:0908.1426 [hep-ph].

7. P. Chiappetta and M. Greco, Nucl. Phys. B199 (1982) 77.
8. G. Pancheri-Srivastava, Y. Srivastava, Phys.Rev. D15 (1977) 2915.

9. Y. I. Dokshitzer, D.I. Dyakonov and S.I. Troyan, Phys. Lett. 79B (1978) 269.

10. G. Parisi and R. Petronzio, Nucl. Phys. B154 (1979) 427.

11. A. Nakamura, G. Pancheri, Y.N. Srivastava, Z. Phys. C21 (1984) 243;

12. A. Corsetti, A. Grau, G. Pancheri, Y. N. Srivastava, Phys. Lett. B382 (1996) 282.

13. Antoni Szczurek, Anna Rybarska, Gabriela Slipek, Phys.Rev. D76 (2007 ) 034001, Antoni Szczurek, Gabriela Slipek, Phys.Rev. D78 (2008)114007.

14. A. M. Polyakov, JETP Lett. 20 (1974) 194.

15. R. M. Godbole, A. Grau, , G. Pancheri, Y. N. Srivastava, Eur. Phys. J. C 63 (2009) 69.

16. M. M. Block and F. Halzen, Phys. Rev. D73 (2006) 054022; E. G. S. Luna and M. J. Menon, arXiv:hep-ph/0105076; J. R. Cudell and O. V. Selyugin, arXiv:hepph/0612046; A. Donnachie and P. V. Landshoff, Phys. Lett. B296 (1992) 227; A. Donnachie and P. V. Landshoff, Phys. Lett. B595 (2004) 393.

17. M. Froissart, Phys. Rev. 123 (1961) 1053.

18. A. Martin, Phys. Rev. 129 (1963) 1432; Nuovo Cimento 42 (1966) 930; A. Martin and F. Cheung, Gordon And Breach Science Publ. , New Yourk 1970. A. Martin, arXiv:0904.3724 [hep-ph]. A. Martin, AIP Conf. Proc. 1105 (2009) 258.

19. L. N. Lipatov, Nucl. Phys.B309 (1988) 379. 